\title{
Contents
}

\section{The Path Ahead}

Jack A. Tuszynski, Nancy Woolf .......................... 1

1.1 Definition and Fundamentals...................... 1

1.1.1 Definition of Consciousness

and the Classical Approach .................... 2

1.1.2 Quantum Theories ........................ 4

1.1.3 Quantum Processing

by Microtubules and Neurocognition ............. 8

1.2 Overview of the Contributions $\ldots \ldots \ldots \ldots \ldots \ldots \ldots \ldots \ldots \ldots$

1.3 New and Notable Developments .................... 17

1.3.1 An Electromagnetic Fingerprint

of Transport Along Microtubules ................ 17

1.3.2 Extrapolations to Mesoscopic and Macroscopic Levels ... 22

1.4 Conclusions................................. 23

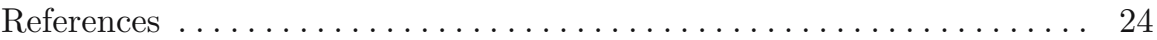

2 Consciousness and Quantum Physics: Empirical Research on the Subjective Reduction of the Statevector

Dick J. Bierman, Stephen Whitmarsh ..................... 27

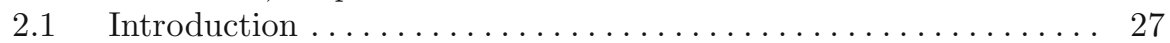

2.1.1 The Measurement Problem ................... 27

2.1.2 Objective Reduction and Consciousness ........... 29

2.1.3 Previous Empirical Work on Subjective Reduction...... 30

2.1.4 Current Investigation ....................... 33

$2.2 \quad$ Experimental Design .............................. 33

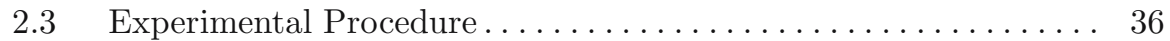

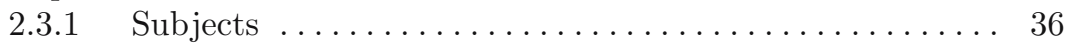

2.3.2 Physiological Measurement ...................... 36

2.3.3 Further Procedure........................... 36

2.4 Data Analysis . . . . . . . . . . . . . . . . . . . . 37

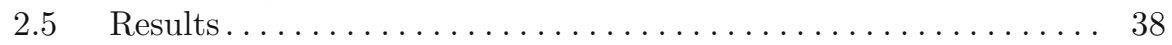

$2.6 \quad$ Conclusions................................... 40

$2.7 \quad$ Further Research .................................. 45

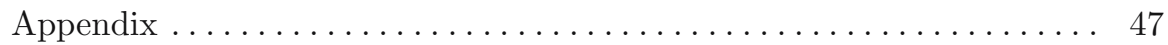

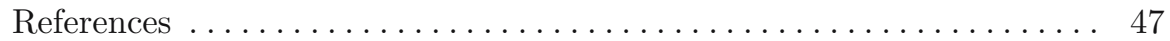




\section{Microtubules in the Cerebral Cortex:}

\section{Role in Memory and Consciousness}

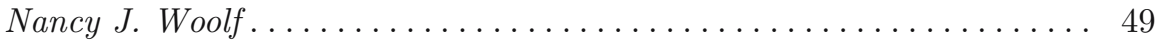

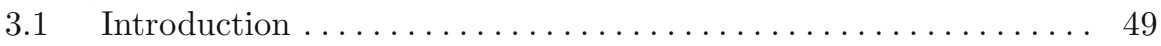

3.1.1 General Features of the Brain . . . . . . . . . . . . 49

3.1.2 Neuronal Assemblies: Patterns of Connection . . . . . . . . 51

3.1.3 Neurons, Synapses and Neurotransmitter Molecules... . . 52

3.2 Functions of Microtubules and MAPs ............. 56

3.2.1 Transport along Microtubules . . . . . . . . . . . . 57

3.2.2 Signal Transduction and Anchoring

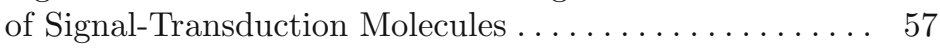

3.3 Learning and Memory: Neuroplasticity vs. Stability . . . . . . . . 65

3.3.1 Synaptic Change: Hebb's Rule Revisited . . . . . . . . . . 66

3.3.2 Microtubules and MAPs in Dendrites

Play a Critical Role in Memory ............. 70

3.3.3 Microtubules Influence Synaptic Efficacy . . . . . . . . . . . 77

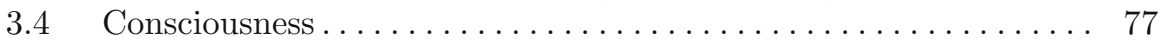

3.4.1 Attention: The Spotlight of Consciousness . . . . . . . . . 78

3.4.2 Waking, Sleeping and Dreaming:

Different Levels of Consciousness . . . . . . . . . . . . 80

3.4.3 Mental Force to Think and Act ............. 81

3.4.4 Consciousness, Memory and Microtubules ......... 83

3.5 Microtubules and Quantum Entanglement:

A Possible Basis for Memory and Consciousness . . . . . . . . . . 85

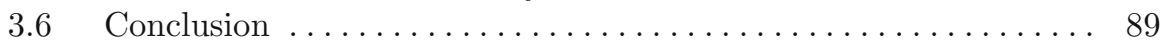

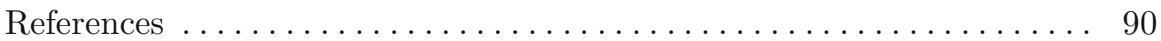

\section{Towards Experimental Tests of Quantum Effects} in Cytoskeletal Proteins

Andreas Mershin, Hugo Sanabria, John H. Miller, Dharmakeerthna

Nawarathna, Efthimios M.C. Skoulakis, Nikolaos E. Mavromatos, Alexadre A. Kolomenskii, Hans A. Schuessler, Richard F. Luduena, Dimitri V. Nanopoulos .......................... 95

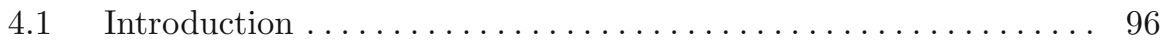

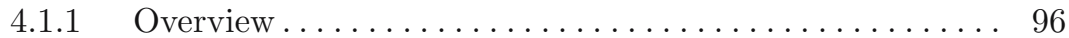

4.1 .2 Tubulin and Microtubules .................. 97

4.1 .3 Motivation .......................... 101

4.2 QED Model of Tubulin and its Implications . . . . . . . . . . . . 102

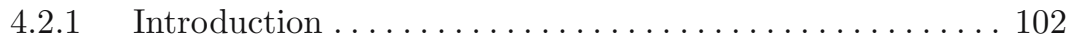

4.2.2 Quantum Coherence in Biological Matter?......... . . 105

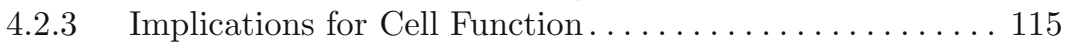

4.2 .4 Conclusions . . . . . . . . . . . . . . . . . . . 120

4.3 Tau Accumulation in Drosophila Mushroom Body Neurons

Results in Memory Impairment . . . . . . . . . . . . . . . . . . . . . . . . . . . 120

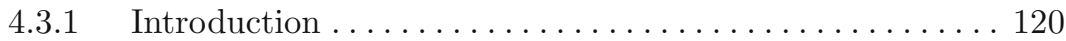


$4.3 .2 \quad$ Drosophila . . . . . . . . . . . . . . . . . . . 121

4.3 .3 Genetic Engineering . . . . . . . . . . . . . . . . 123

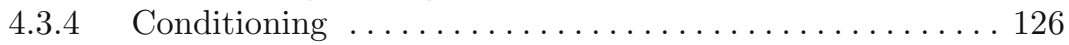

4.3 .5 Controls ............................. 128

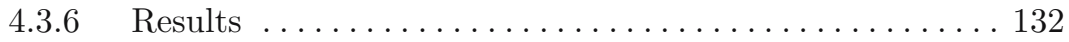

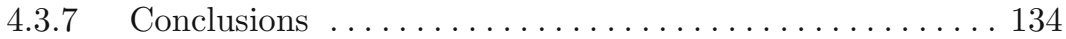

4.3 .8 Discussion ............................. 134

4.4 Refractometry, Surface Plasmon Resonance, and Dielectric

Spectroscopy of Tubulin and Microtubules . . . . . . . . . . . 136

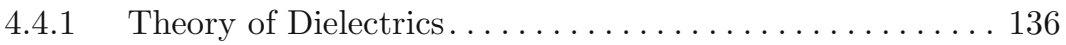

4.4 .2 Optics . . . . . . . . . . . . . . . . . . . 141

4.4.3 Surface Plasmon Resonance $(\mathrm{SPR}) \ldots \ldots \ldots \ldots \ldots . \ldots \ldots$

4.4 .4 Dielectric Spectroscopy . . . . . . . . . . . . . . . . . . 153

4.5 Emerging Directions of Experimental Tests

of the Quantum Consciousness Idea . . . . . . . . . . . . . . . 159

4.5.1 Entanglement . . . . . . . . . . . . . . . . . . . 159

4.5.2 Molecular Electronics . . . . . . . . . . . . . . . 160

4.5.3 Proposed Further Research . . . . . . . . . . . . . . . . 160

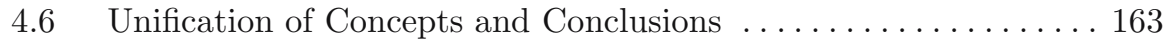

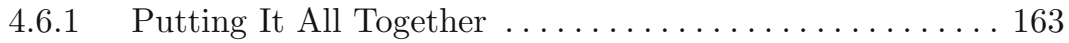

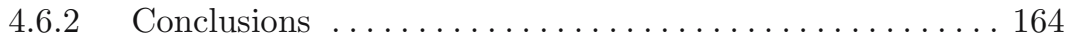

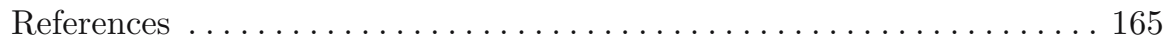

\section{Physicalism, Chaos and Reductionism}

Alwyn Scott ................................... 171

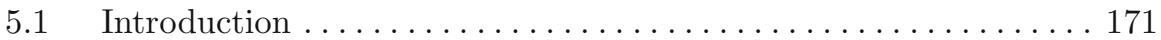

5.2 Quantum and Classical Dynamics . . . . . . . . . . . . . 172

5.3 What Are Classical Nonlinear Phenomena? . . . . . . . . . . . . . . . 173

5.4 The Biological and Cognitive Hierarchies . . . . . . . . . . . . . . . . . . 174

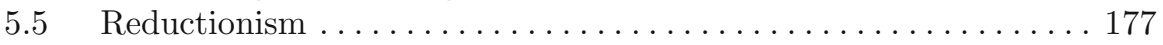

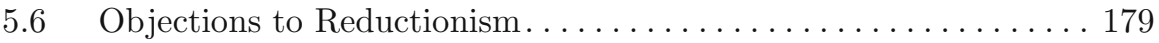

5.6.1 Constructionism versus Reductionism . . . . . . . . . . 179

5.6 .2 Immense Numbers of Possibilities . . . . . . . . . . . . . 180

5.6 .3 Sensitive Dependence on Initial Conditions . . . . . . . . . 181

5.6 .4 The Nature of Causality . . . . . . . . . . . . . 181

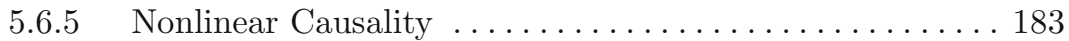

5.6 .6 The Nature of Time . . . . . . . . . . . . . . . 184

5.6 .7 Downward Causation ....................... 184

5.6 .8 Open Systems . . . . . . . . . . . . . . . . . . . . 185

5.6 .9 Closed Causal Loops. . . . . . . . . . . . . . . . . . 186

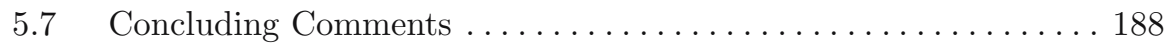

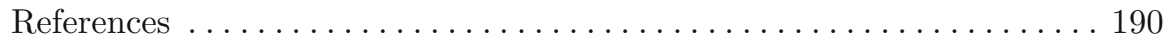




\section{Consciousness, Neurobiology and Quantum Mechanics: The Case for a Connection}

Stuart Hameroff . . . . . . . . . . . . . . . . . . . . . . . . . . . . . . . . . . . . . 193

6.1 Introduction: The Problems of Consciousness . . . . . . . . . . . . . 193

6.2 Time and Consciousness . . . . . . . . . . . . . . . . . . 197

6.2.1 Is Consciousness Continuous

or a Sequence of Discrete Events? . . . . . . . . . . . 197

6.2.2 The Timing of Conscious Experience . . . . . . . . . . 198

6.2.3 Taking Backward Time Referral Seriously . . . . . . . . . . 202

6.3 The Neural Correlate of Consciousness . . . . . . . . . . . . . . . . 206

6.3.1 Functional Organization of the Brain . . . . . . . . . 206

6.3.2 Cerebral Cortex and Neuronal Assemblies . . . . . . . . . . . 208

6.3.3 Axons and Dendrites ..................... 208

6.3 .4 Neural Synchrony . . . . . . . . . . . . . . . . . . . . 212

6.3.5 Gap-Junction Assemblies - "Hyperneurons" . . . . . . . . 215

6.3.6 The Next NCC Frontier -

Neuronal Interiors and the Cytoskeleton . . . . . . . 216

6.4 The Neuronal Cytoskeleton . . . . . . . . . . . . . . . . 217

6.4.1 Microtubules and Networks inside Neurons . . . . . . . . . . . 217

6.4 .2 Microtubule Automata .................... 220

6.4.3 Protein Conformational Dynamics -

Nature's Bits and Qubits ................... 224

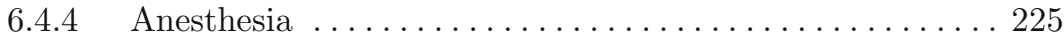

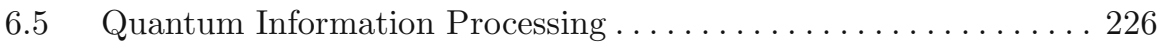

6.5.1 Quantum Mechanics ..................... 226

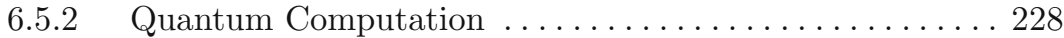

6.5.3 Quantum Computing with Penrose OR ........... 229

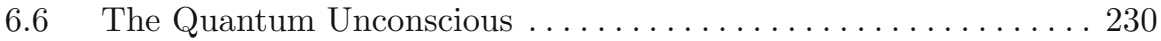

6.7 Quantum Computation in Microtubules - The Orch OR Model. . 232

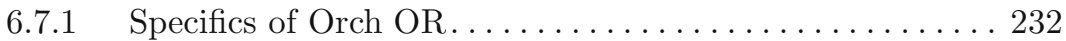

6.7 .2 Decoherence ........................... 235

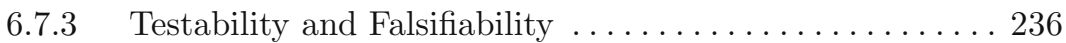

6.8 Applications of Orch OR to Consciousness and Cognition . . . . . . 236

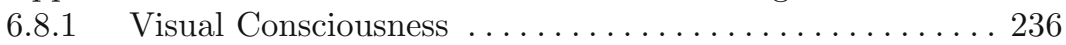

$6.8 .2 \quad$ Volition and Free-Will ................... 238

6.8.3 Quantum Associative Memory . . . . . . . . . . . . . . . 239

6.8.4 The Hard Problem of Conscious Experience . . . . . . . . 239

6.8.5 What is Consciousness? . . . . . . . . . . . . . . 240

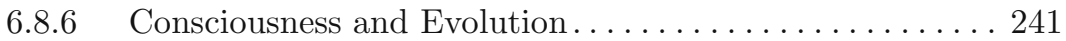

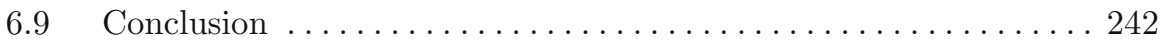

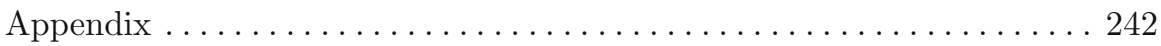

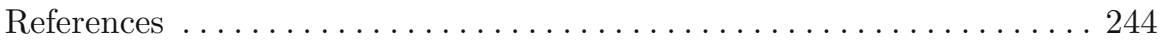


7 Life, Catalysis and Excitable Media:

A Dynamic Systems Approach to Metabolism and Cognition

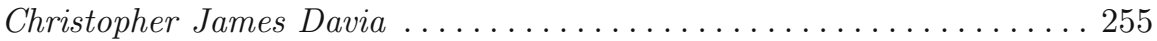

7.1 Life and Robustness . . . . . . . . . . . . . . . . . . . 255

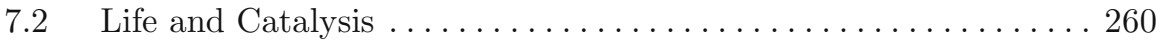

7.3 Catalysis, Traveling Waves and Excitable Media . . . . . . . . . . . 271

7.4 The Brain as an Excitable Medium . . . . . . . . . . . . . 274

7.5 Conclusion .............................. 288

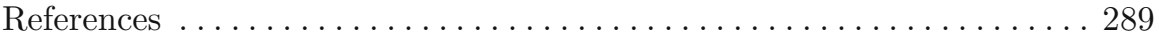

\section{The Dendritic Cytoskeleton as a Computational Device:} An Hypothesis

Avner Priel, Jack A. Tuszynski, Horacion F. Cantiello.............. 293

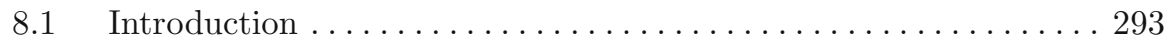

$8.1 .1 \quad$ Neurobiological Introduction . . . . . . . . . . . . . . . 293

8.1 .2 Neuro computational Introduction . . . . . . . . . . . . . . 297

8.1.3 Dendritic Channel Function . . . . . . . . . . . . . . . 299

8.1.4 Actin-Microtubule Cytoskeletal Connections . . . . . . . . . 299

8.2 C-Termini in Microtubules . . . . . . . . . . . . . . . . . . 301

8.2.1 Potential Configurations of Microtubular C-Termini . . . 303

8.2.2 Dynamic Model of the C-Termini............... . 305

8.2 .3 Ionic Wave Propagation along MAP2 . . . . . . . . . 306

8.3 Ion Waves along Actin Filaments . . . . . . . . . . . . . . . 308

8.3.1 Ionic Condensation along the Actin Filament . . . . . . . 308

8.3.2 Electrical Modeling of Actin . . . . . . . . . . . . . 309

8.3.3 Implications of Actin Filament's Electrical Activity . . . . 312

8.4 Dendritic Cytoskeleton Computation - Vision of Integration . . . 313

8.4.1 MTN Control of Synaptic Plasticity,

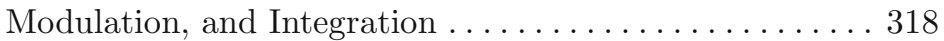

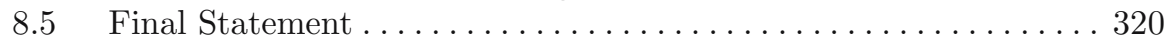

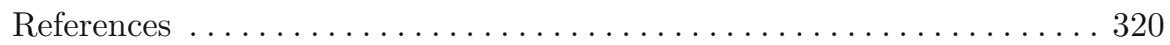

\section{Recurrent Quantum Neural Network and its Applications}

Laxmidhar Behera, Indrani Kar, Avshalom C. Elitzur. . . . . . . . . . 327

9.1 Intelligence - Still Ill-Understood . . . . . . . . . . . . . . . 327

9.2 Intelligent Filtering - Denoising of Complex Signals . . . . . . . . . 328

9.2.1 RQNN Architecture used for Stochastic-Filtering . . . . . . 329

9.2.2 Integration of the Schrödinger Wave Equation . . . . . . . . . . 331

9.2 .3 Simulation Results I . . . . . . . . . . . . . . 333

9.3 A Comprehensive Quantum Model of Intelligent Behavior . . . . . 337

9.4 RQNN-based Eye-Tracking Model . . . . . . . . . . . . . . . . 338

9.4.1 A Theoretical Quantum Brain Model .............. 338

9.4.2 An Eye-Tracking Model using RQNN

with Nonlinear Modulation of Potential Field . . . . . . . 339

9.4 .3 Simulation Results II . . . . . . . . . . . . . . . . . . 342 


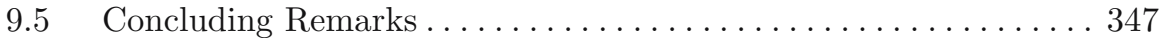

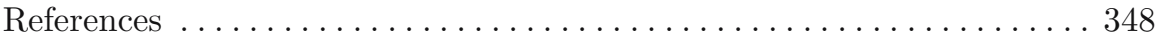

10 Microtubules as a Quantum Hopfield Network

Elizabeth C. Behrman, K. Gaddam, J.E. Steck, S.R. Skinner ......... 351

10.1 Introduction . . . . . . . . . . . . . . . . . . . . . . 351

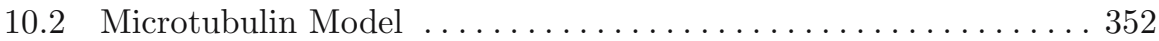

10.3 Hopfield Model. . . . . . . . . . . . . . . . . . . . . . . . . . . . . . 354

10.4 Quantum Model. . . . . . . . . . . . . . . . . . . . . . . . . . . 355

10.5 Quantum Hopfield Network . . . . . . . . . . . . . . . . . . 358

10.6 QHN as Information Propagator for a Microtubules Architecture 360

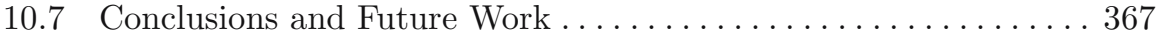

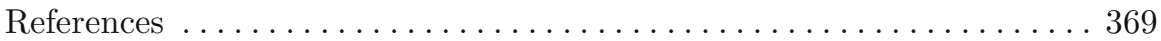

\section{Consciousness and Quantum Brain Dynamics}

Gordon Globus ........................................ 371

11.1 Deconstruction . . . . . . . . . . . . . . . . . . . . 371

11.2 Quantum Brain Dynamics . . . . . . . . . . . . . . . . . . 373

11.3 Hermitean Dual-Mode Quantum Brain Dynamics . . . . . . . . . . . 375

11.4 Non-Hermitean Dual-Mode Quantum Brain Dynamics . . . . . . . 376

11.5 Application to Mathematics: The Riemann Hypothesis . . . . . . . . 377

11.6 Monadological Implications of Non-Hermitean Dual-Mode QBD . 381

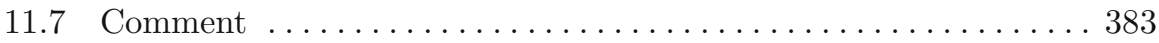

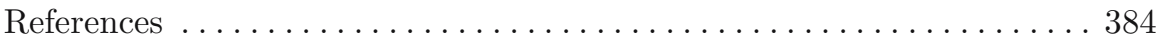

\section{The CEMI Field Theory:}

\section{Seven Clues to the Nature of Consciousness}

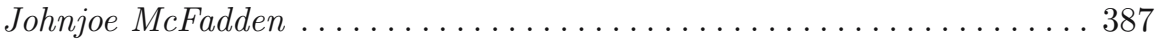

12.1 Why Do we Need a Theory of Consciousness? . . . . . . . . . . . . 387

12.2 Field Theories of Consciousness . . . . . . . . . . . . . . . . . 393

12.3 The Brain's Electromagnetic Field . . . . . . . . . . . . . . . . . . . . . . . . . 394

12.4 The Influence of the Brain's Electromagnetic Field

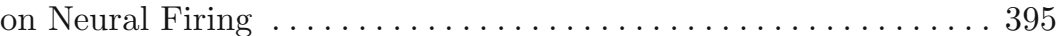

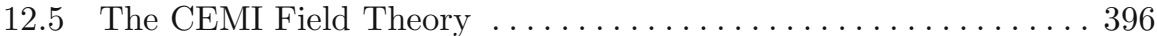

12.6 Why don't External Fields Influence our Minds? . . . . . . . . . . . 397

12.7 Does the CEMI Field Theory Account for the Seven Clues to the Nature of Consciousness? . . . . . . . . . . . . . . . . . . . . 398

12.8 A Last Word, Concerning Quantum Theories of Consciousness . . 401

12.9 Conclusions and the Way Forward ... . . . . . . . . . . . . . 404

References .................................... 404

\section{Quantum Cosmology} and the Hard Problem of the Conscious Brain

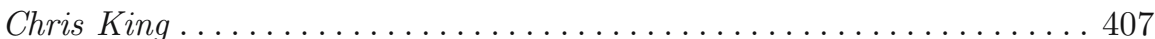

13.1 Subject-Object Complementarity and the Hard Problem . . . . . . 407 
13.2 Wave-Particle Complementarity, Uncertainty

and Quantum Prediction ......................... 410

13.3 Two-Timing Nature of Special Relativity ................ 415

13.4 Reality and Virtuality:

Quantum Fields and Seething Uncertainty ............... 416

13.5 The Spooky Nature of Quantum Entanglement.............. 417

13.6 Quantum Match-Making:

Transactional Supercausality and Reality .............. 420

13.7 Exploring the "Three Pound Universe" ................... 423

13.8 Chaos and Fractal Dynamics as a Source

of Sensitivity, Unpredictability and Uncertainty ........... 428

13.9 Classical and Quantum Computation, Anticipation and Survival. 430

13.10 The Cosmic Primality of Membrane Excitation ............. 433

13.11 Chaotic Excitability and Quantum Sensitivity

as a Founding Eucaryote Characteristic ............... 437

13.12 Models of the Global-Molecular-Quantum Interface . . . . . . . . 440

13.13 Quantum Mind and Transactional Supercausality .......... 442

13.14 Complementarity and the Sexuality of Quantum Entanglement. . 448

13.15 The Hard Problem: Subjective Experience, Intentional Will and Quantum Mind Theories ........................ 449

13.16 Consciousness and Neurocosmology . . . . . . . . . . . . . . 451

References ................................. 454

14 Consciousness and Logic in a Quantum Computing Universe

Paola Zizzi..................................... 457

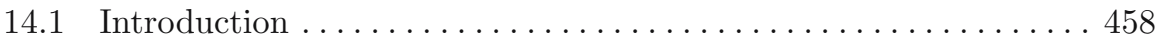

14.2 The "Big Wow" ............................. 459

14.3 How the "Big Wow" Drove Human Minds ................. 461

14.3.1 Entanglement with the Environment.............. 463

14.3.2 Holography and Cellular Automata ............... 463

14.4 Consciousness and Tubulins/Qubits ................... 464

14.5 Consciousness Arises in the "Bits Era" ................. 465

14.5.1 The Boolean Observer ....................... 465

14.5.2 The Analogy ......................... 466

14.6 The Double Logic of the Observer Inside a Quantum Universe . 467

14.7 IT from Qubit: The Whole Universe as a Quantum Computer ... 468

14.8 Quantum Minds and Black - Hole Quantum Computers

in a Quantum Game ............................ 469

14.9 Qualia and Quantum Space-Time ................. 470

14.10 Mathematical Intuition and the Logic of the Internal Observer . 473

14.11 The Self ............................... 475

14.11.1 The Self and the Mirror Measurement .......... 475

14.11 .2 Nonself ............................ 476 
XII Contents

14.11.3 The Universal Self: The Universe and the Mirror . . . . . . 476

14.11.4 The Universal Self: The Mathematical Truth. . . . . . . . . . 477

14.12 Conclusion . . . . . . . . . . . . . . . . . . . . . . . . . . . . 477

References ................................. 479

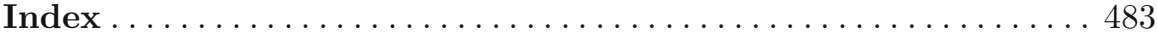

